\title{
Cambios en la organización económico-espacial de la fruticultura en territorios de La Araucanía, Chile
}

\section{Changes in economic and spatial organization of fruit growing in territories of La Araucanía, Chile}

\author{
Miguel Antonio Escalona-UlloA* \\ Fernando Andrés Peña-Cortés* \\ Gonzalo Daniel Rebolledo-Castro* \\ Pío Iván Antonio Basso-AldeA**
}

\begin{abstract}
The article discusses the potential expansion of fruit growing in La Araucania region, identifying territories that have an aptitude for future development. A historical analysis is carried out and a spatial model to define suitable areas is produced. The outcomes allow recognizing the existence of two specific areas: north Malleco, formed by Angol, Renaico and Collipulli; and north Cautin, integrated by the districts of Freire, Pitrufquén, Gorbea, Loncoche and Villarrica. In these spaces it is possible to further expand the activity but it requires to improve the infrastructure and to incorporate a territorial approach to regional economic development.
\end{abstract}

Keywords: Territory, territorial development, spatial organization.

\section{Resumen}

El artículo aborda el potencial de expansión de la actividad frutícola en la región de La Araucanía, identificando territorios que presentan aptitud para su desarrollo futuro. Se realiza un análisis histórico y se elabora un modelo espacial para definir áreas aptas, el resultado permite reconocer la existencia de dos territorios específicos: Malleco Norte, conformados por Angol, Renaico y Collipulli, y Cautín sur, integrado por las comunas de Freire, Pitrufquén, Gorbea, Loncoche y Villarrica, en estos espacios es posible expandir aún más la actividad pero se requiere mejorar la infraestructura e incorporar un enfoque territorial al desarrollo productivo regional.

Palabras claves: Territorio, desarrollo territorial, organización espacial. uct.cl

*Universidad Católica de Temuco. Chile. Correos-e: mescalon@uct.cl, fpena@uct.cl, grebolle@

** Secretaría Regional Ministerial de Agricultura. Región de La Araucanía. Correo-e: ibasso@ minagri.cl 


\section{Introducción}

La globalización ha sido el catalizador de la integración de diversos espacios locales de América Latina dentro de una compleja y desigual red de interconexiones territoriales (Barton et al., 2007). Este proceso ha producido cambios sustanciales en la estructura de la actividad económica, con repercusiones sobre la organización del territorio (Cuervo, 2006). Estos cambios pueden explicarse a través de postulados provenientes de la geografía económica, los cuales señalan que en la actualidad existe una gran diversidad de formas de aglomeración económica en espacios geográficos (Fujita y Krugman, 2004). Para ello es necesario entender cómo las economías se vuelven complejas y cómo esta concepción se inscribe en el pensamiento evolucionista (Pike et al., 2009). El aumento de la complejidad asociado a cambios rápidos alcanza su máxima expresión en las grandes aglomeraciones urbanas, cuyas economías y territorios han experimentado con intensidad los cambios estructurales que marcaron la transición hacia nuevas formas de organización del sistema (Peńa y Escalona, 2009).

En la actualidad existe una estrecha relación entre la globalización y el territorio, es por ello que Friedman (2006) plantea que el terreno de juego de la competencia a escala global se está nivelando. Esto permite que el sistema territorial (Gómez-Orea, 2007) se encuentre tensionado por múltiples y coexistentes vías (Costa-Filho, 1997), generando también oportunidades móviles y cambiantes. En este contexto, la organización espacial de la producción cambia y se transforma, así las estrategias territoriales de las empresas y las estrategias económicas de las ciudades y regiones están condicionadas a este cambio (Vázquez-Barquero, 2006).

$\mathrm{Al}$ respecto, Chile ha utilizado acuerdos comerciales bilaterales y multilaterales como forma de promover la exportación de su modelo de desarrollo implementado desde la década de 1980. Es el único país de América que ha negociado con éxito acuerdos de libre comercio o de complementación económica con economías centrales más importantes (EE.UU. y la UE), y con los principales mercados emergentes en las Américas y este de Asia (como China en 2006, Corea, México y el Mercado Común Centroamericano) (Barton et al., 2007). Sin embargo, las regiones del país no tienen una plataforma común para elevar los indicadores de competitividad, porque sus economías están basadas en distintas dotaciones y combinaciones de factores de producción, conocimiento y capital social, principalmente. Consecuentemente, es posible distinguir que las estrategias globales o sectoriales que se siguen no son iguales, porque las realidades y expectativas son diferentes entre los agentes de producción, transformación y comercialización. 
Las dinámicas territoriales actuales requieren comprender que cada territorio tiene sus particulares características, un carácter heterogéneo de los sectores rurales e importantes vínculos con lo urbano. Para que un territorio se desarrolle debe fortalecer los lazos con sectores de la economía que le sirvan de motor para consolidar el crecimiento económico, pero además, se requiere un fuerte desarrollo institucional (Pacheco, 2001; Etcheverri, 2003; Schejtmann y Berdegue, 2004; Fonte, 2006; Ranaboldo y Schejtmann 2009). Por este motivo los procesos endógenos que se registran en pequeñas unidades territoriales y asentamientos humanos son capaces de promover el dinamismo económico y la mejora en la calidad de vida de la población (Boisier, 2006). Esta construcción de capacidades posibilita los procesos de organización a través de la generación de conocimientos intangibles y el aprovechamiento de los recursos endógenos territorialmente emplazados a partir de la sinergia conjunta de actores públicos y privados (Fernández et al., 2009). Esta referencia que se hace al territorio no es genérica, es decir, no se refiere al ámbito de distribución espacial de las actividades productivas, sino a los correspondientes entornos de la vida cotidiana en los distintos territorios, en los cuales los elementos socioculturales y de identidad se entrelazan con las actividades económicas locales influenciándose de forma recíproca (Albuquerque, 2006).

La dinámica económica y productiva de la región de La Araucanía en Chile está determinada por sus características como territorio de Frontera. En su proceso histórico de ocupación y organización espacial destaca la existencia del fuerte como elemento construido, posteriormente, comienza un desarrollo de poblamiento y ocupación del territorio, consolidándose la cuenca del río Cautín como eje de partida del movimiento económico regional, luego la llegada del ferrocarril, como un nuevo concepto de desplazamiento consolida definitivamente la fundación de los asentamientos (IGM, 1986; Jara y Valeria, 1987) (figura II).

El proceso de ocupación parte por el sector norte de la región (provincia de Malleco), este territorio compone el antiguo Granero de Chile, donde predominaban los cultivos de trigo y cereales. La aptitud triguera de estas tierras ha permitido la obtención de destacados rendimientos, situación, que es posible observar en la comuna de Traiguén, la cual conservó por mucho tiempo condiciones de fertilidad con unos rendimientos de 13 quintales por hectárea (qqm/ha); al igual que Temuco y Lautaro con un rendimiento de $14 \mathrm{qqm} / \mathrm{ha}$, cifras que de acuerdo con la Dirección General de Estadísticas y Censos Agropecuarios de 1929 y 1935 aportaban 30.7 \% de la producción nacional (IGM, 1986; Bengoa, 1996; Rosenblitt y Naser, 2005). 
Si se observa la información oficial de los censos agropecuarios desde 1965 hasta 2007, es posible observar una tendencia en la disminución de los cereales y chacras, aun cuando representan sobre $40 \%$ de la superficie sembrada. Situación similar ocurre con las plantaciones forestales que aumentan de 11\% (1964) a 49.6\% para 1997; respecto a la plantas forrajeras, las cuales están vinculadas con la actividad agropecuaria, se mantienen alrededor de $20 \%$. Por su parte, los frutales aumentan entre 1975 a 1997 en 50\%, en menor medida las viñas presentan una tendencia similar (cuadro 1).

En el presente trabajo, se analizan las actividades productivas con implicancia territorial, particularmente el caso de la fruticultura. Cabe señalar que el consumo mundial de frutas ha tenido un crecimiento sostenido, con una tasa anual de $2.5 \%$ entre 1995 y 2005 , correspondiente a un aumento del consumo, desde 395 millones de toneladas en 1995 a 493 millones de toneladas en 2005 . Este crecimiento fue similar al consumo a nivel mundial de carne, sin embargo, fue superior al crecimiento del consumo de vegetales (2.4\%) y de pescados (0.1\%) (вСG, 2007).

En Chile, el sector frutícola está posicionándose cada vez más como uno de los clusters con mayor potencial de crecimiento, cuya producción total representa 2.6\% del PIB nacional. Según ODEPA (2007), la superficie plantada con especies frutales aumentó a más del doble en diez años, pasando de 79.500 hectáreas en 1979 a 171.400 en 1989, para luego aumentar en 2004 a 221.915 hectáreas, lo que significó una tasa de crecimiento promedio anual de $1.7 \%$ en el periodo. Este crecimiento ha sido aún mayor ya que la superficie nacional plantada con especies frutícolas alcanza las 324.279 hectáreas, lo que significa que entre 2004 y 2007 el sector frutícola a nivel nacional experimentó un crecimiento de 46\% (INE, 2007).

El valor de los envíos de fruta fresca chilena al mundo experimentó un alza de $16 \%$ en la temporada 2007-2008, totalizando 3,197,300 millones de dólares. El volumen exportado registró una caída moderada de 2\% en ese periodo. La proyección para la temporada 2008-2009 indica que los niveles de producción y de calidad tendrían una mejora, debido a que la crisis financiera internacional promoverá una selectividad por frutas de consumo más masivo y los consumidores serán atraídos por ofertas y precios atractivos, tanto en el mercado norteamericano como en el europeo, lo cual traerá bajas de precios, sobre todo en los productos Premium de mayor valor, como los arándanos y las cerezas (ODEPA, 2009).

La Araucanía en los últimos cinco años ha mostrado que en varios territorios y zonas específicas es posible la introducción de nuevas técnicas y tecnologías, para sacar provecho de óptimos biológicos y económicos. En este contexto, la articulación de una economía territorial implica el 


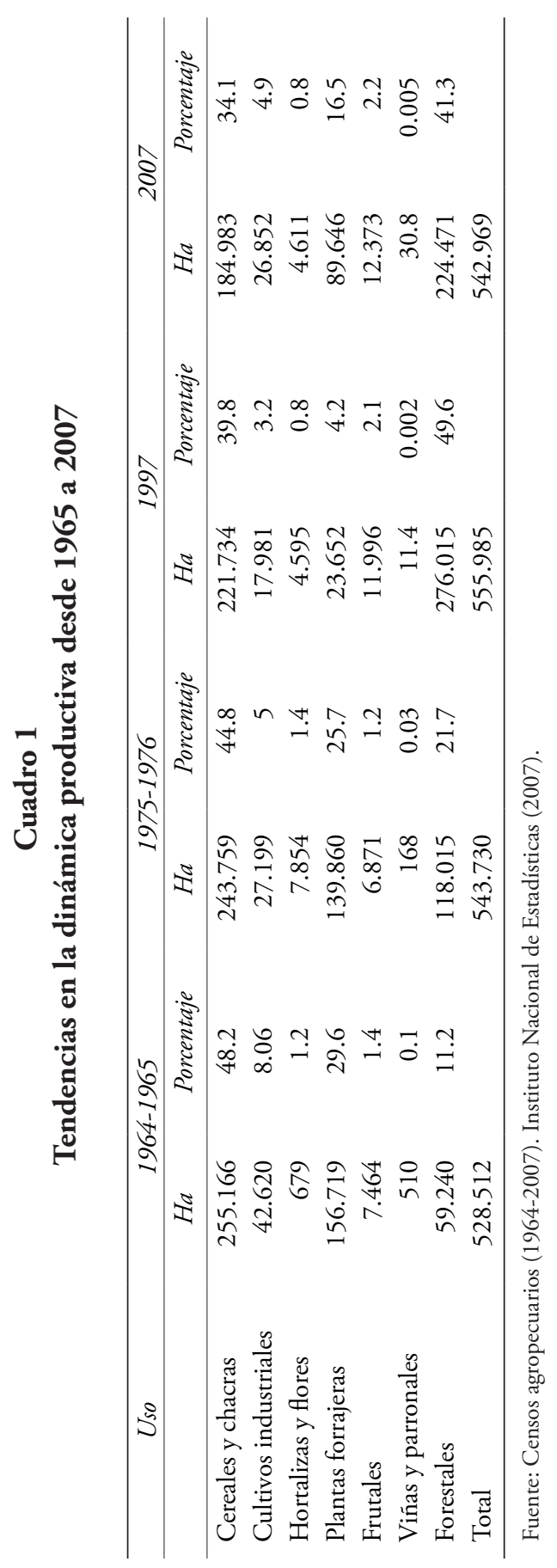


reconocimiento de la competitividad proveniente de sus ventajas competitivas y comparativas, las cuales se desarrollan a partir de las relaciones de los diferentes eslabones de la cadena productiva (Seremi de Agricultura, 2007). Unido a ello, el desarrollo de cadenas de valor basadas en rubros tradicionales o innovadores, que han ajustado o están ajustando sus procesos y estrategias de encadenamiento y de búsqueda y posicionamiento de mercados: berries (frambuesas, frutillas, arándanos), así como, avellano europeo, manzanos, cerezos, ciruelas, peras, kiwis, moras cultivadas, hortalizas, flores y otros, forman parte del patrimonio productivo y de las realidades de desarrollo de amplias zonas, que pueden fortalecerse para beneficio de más actores, especialmente de pequeños campesinos organizados con capacidad de gestión, que esperan y demandan apoyo para mejorar o establecer nuevas iniciativas.

En este contexto, la investigación que a continuación se presenta aborda el potencial de expansión para la actividad frutícola en la región de La Araucanía, esto con el objeto de identificar aquellos territorios que presentan aptitud para el desarrollo de esta actividad y aquellos que a futuro pueden ser incorporados, para así dotarlos con infraestructura vial y conectividad, equiparlos con una cantidad adecuada de servicios y promover estrategias de intervención tanto públicas como privadas.

\section{Metodología}

Para la determinación del cambio en la organización espacial se identificó el nivel de expansión de la fruticultura a partir de la elaboración de un modelo espacial para luego comparar los resultados con estadísticas de producción e inversión.

La construcción del modelo se realiza sobre la base del reconocimiento del grado de satisfacción de los requerimientos de cada tipo de uso de suelo o actividad definida por un uso y una unidad territorial, para la posterior planificación y gestión del conjunto de usos en la totalidad del territorio (Santé y Crecente, 2005), para ello se consideraron procesos de aplicación Multicriterio con un Sistema de Información Geográfica (SIG) y panel de expertos (Gómez y Barredo, 2006) y diversas operaciones de álgebra de mapas sobre modelo de datos raster con pixel de $1000^{*} 1000 \mathrm{~m}$, utilizando Spatial Analyst y Model Builder de ArcView ${ }^{\text {TM }}$ 3.2 y ArcGis $^{\text {тм }} 8.2$.

El modelo fue construido con base en dos criterios: (1) aptitud física para el emplazamiento de la actividad frutícola y (2) restricciones para su emplazamiento (cuadro 2). Para este último criterio se consideraron valores umbrales que se expresan en forma binaria donde 1 (uno) es igual a la posibilidad de realización de la actividad y 0 es igual a la imposibili- 
dad de realizar la actividad; de esta forma el factor discrimina todas las áreas con altitudes menores a $500 \mathrm{msnm}$, división predial superior a las 10 hectáreas y pendientes menores a $20 \%$, como áreas que permiten el desarrollo de la actividad.

\section{Cuadro 2}

Factores utilizados en la elaboración del Modelo de Aptitud para el emplazamiento de la actividad frutícola

\begin{tabular}{lc}
\hline \multicolumn{1}{c}{ Factores } & Ponderación \\
\hline Aptitud física de emplazamiento (Afem) & \\
Infraestructura de riego extrapredial & 30 \\
Red hídrica & \\
Capacidad de uso de suelo & 20 \\
Humedad relativa & 15 \\
Periodo libre de heladas & 15 \\
Accesibilidad & 20 \\
Restricciones (Res)* & \\
Altitud & \\
División predial & \\
Pendiente & \\
\hline
\end{tabular}

Fuente: Elaboración propia.

La expresión algebraica del modelo es la siguiente:

$$
A E M=\sum\left[\left(\sum V x i * P i\right) *(V r 1 * V r 2 * V r 3)\right]
$$

Donde:

$A E M=$ Aptitud para el emplazamiento de la actividad frutícola.

$V x i=$ Variables aptitud física según cuadro 2.

$V r=$ Variables de restricción.

El resultado del modelo surge de la integración de la Aptitud Física con las Restricciones, la cual deja fuera aquellos territorios con menos potencial diferenciándolos de aquellos que presentan mayor aptitud.

El segundo paso consistió en comparar los resultados del modelo con información respecto a valores brutos de producción agropecuaria (BCG, 2007) y agroindustrial (ODEPA, 2007), la superficie plantada (INE, 2007), la mano de obra asociada, las inversiones respecto a ella y la innovación, entre otros factores de competitividad. 


\section{Resultados y discusión}

El análisis espacial desarrollado permitió definir dos áreas geográficas que representan sobre 20\% de la superficie regional: Angol-Renaico-Collipuli, denominado Malleco Norte, éste abarca una superficie de 279.518 hectáreas, equivalentes a $8.73 \%$ de la región, el cual fue uno de los primeros territorios en consolidar una identidad frutícola, y LoncocheVillarrica-Los Laureles-Freire, conocido como Cautín Sur, que abarca una superficie de 448.769 hectáreas, equivalentes a $14 \%$ de la superficie regional, representado por un gran número de proyectos con enfoque hacia mercados externos. Estos resultados permiten observar el nivel de cambio de la organización espacial en torno a la actividad frutícola, que se refleja en las áreas favorables para el desarrollo de la actividad concentrada en la depresión central de la región, abarcando el eje asociado a la ruta 5 sur, desde Angol-Renaico por el norte hasta Freire-Gorbea-Pitrufquén por el sur, específicamente, los sectores de mayor aptitud para el desarrollo de la fruticultura abarcarían 53.000 hectáreas (figura I), de los cuales La Araucanía ocupa en la actualidad sólo 22\% (12.000 hectáreas).

Los territorios identificados Malleco Norte y Cautín Sur abarcan 23\% de la superficie regional y en ellos se concentran $52.5 \%$ de las plantaciones de frutales. En estos espacios es posible expandir aún más la superficie plantada debido a características intrínsecas que presenta el territorio, si a ello sumamos el efecto del cambio climático, estamos en presencia de una nueva tendencia en la configuración de la matriz productiva regional.

De esta manera, a nivel regional estos espacios presentan un mayor potencial de expansión, dado tanto por sus condiciones físicas para el desarrollo de la actividad frutícola como por los niveles de producción e inversión. Si se analizan los mayores valores brutos de producción frutícola para 2006 destacan los territorios de Malleco Norte y Cautín Sur, debido a que en estas comunas se han instalado empresas exportadoras dedicadas a la producción primaria como a la producción agroindustrial en lugares específicos, destacan Frutícola Angol y Sociedad Huertos Collipulli s.A, entre otras, asociadas al primer territorio, y San Jose Farms s.A., Patagonia Food, asociadas al segundo territorio. Esto ha originado el surgimiento de cadenas de valor que requieren completar sus distintos eslabones (producción, transformación y comercialización) con elementos del entorno.

En este contexto, Malleco Norte y Cautín Sur (figura II) presentan una vocación productiva que ha permitido establecer una importante tasa de emprendimientos y desafíos importantes en aspectos de formación de capital humano, industrialización de la producción primaria para completar un sistema de valor en la cadena frutícola, y la provisión de nume- 


\section{Figura I}

\section{Representación regional del modelo de aptitud para el emplazamiento de la actividad frutícola}

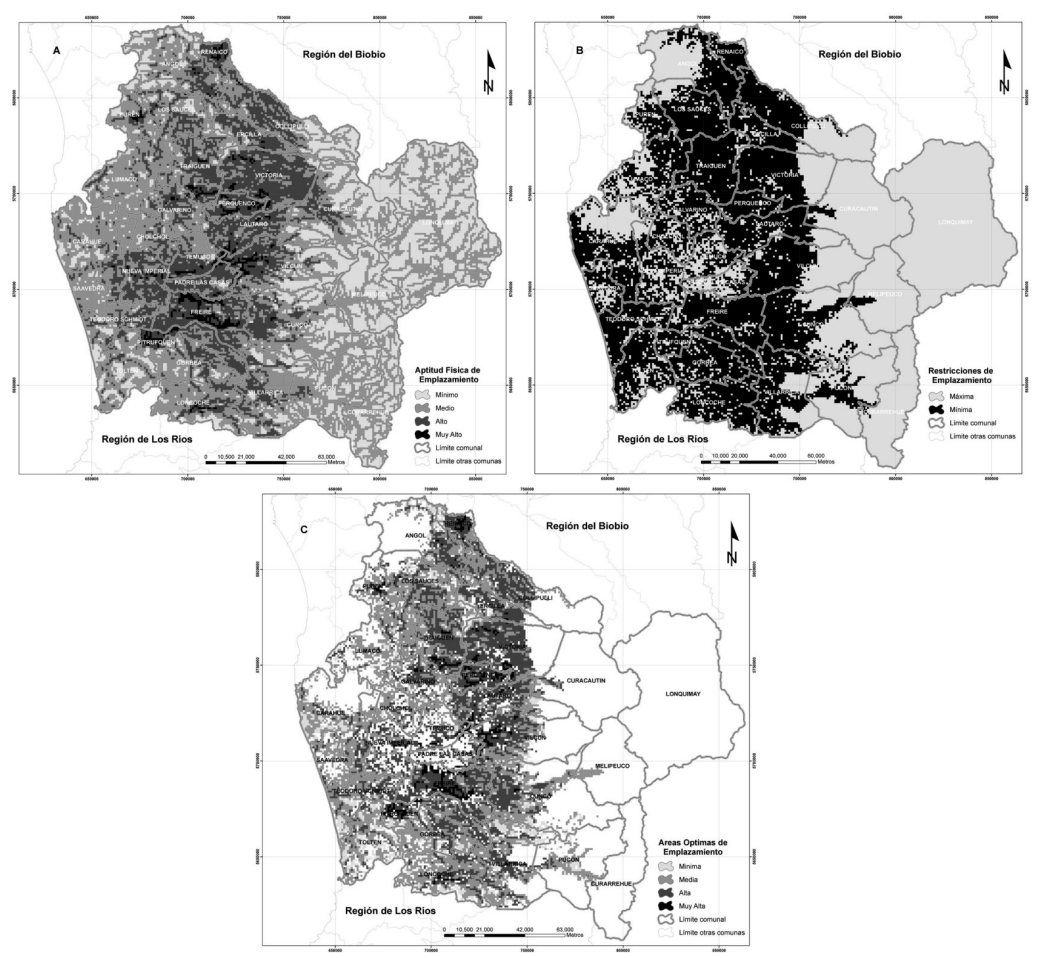

A: Modelo de Aptitud Física del territorio; B: Modelo de Restricciones del territorio; C: Areas óptimas para el emplazamiento.

Fuente: Elaboración propia.

rosos servicios a proyectos que demandarán la consolidación de un spin off nuevo; un factor importante lo constituye la visión de algunos empresarios innovadores que se han instalado con empresas dedicadas tanto a la producción de fruta fresca como congelados, entre las cuales destacan: Frutícola Olmué s.A., Sociedad Agrícola Sunberry Ltda, Sociedad Agrícola Franparque Ltda.; Southern Cherry s.A., entre otras (ARDP, 2008).

Sin embargo, si se proyecta el Valor Bruto de la Producción Primaria (VBPP) al 2014, la fruta se transformaría en el sector de mayor representación regional, seguida del trigo y la leche (figura III). Esto implica la necesidad de prepararse para este nuevo escenario con el fin de mejorar la competitividad de este rubro a nivel nacional y global. Este cambio que está sucediendo en la región es posible cuantificarlo a través del estudio realizado por ODEPA (2007), el cual plantea que en la representación regional de los sectores para el 2006 predomina el trigo con $42.4 \%$ del 


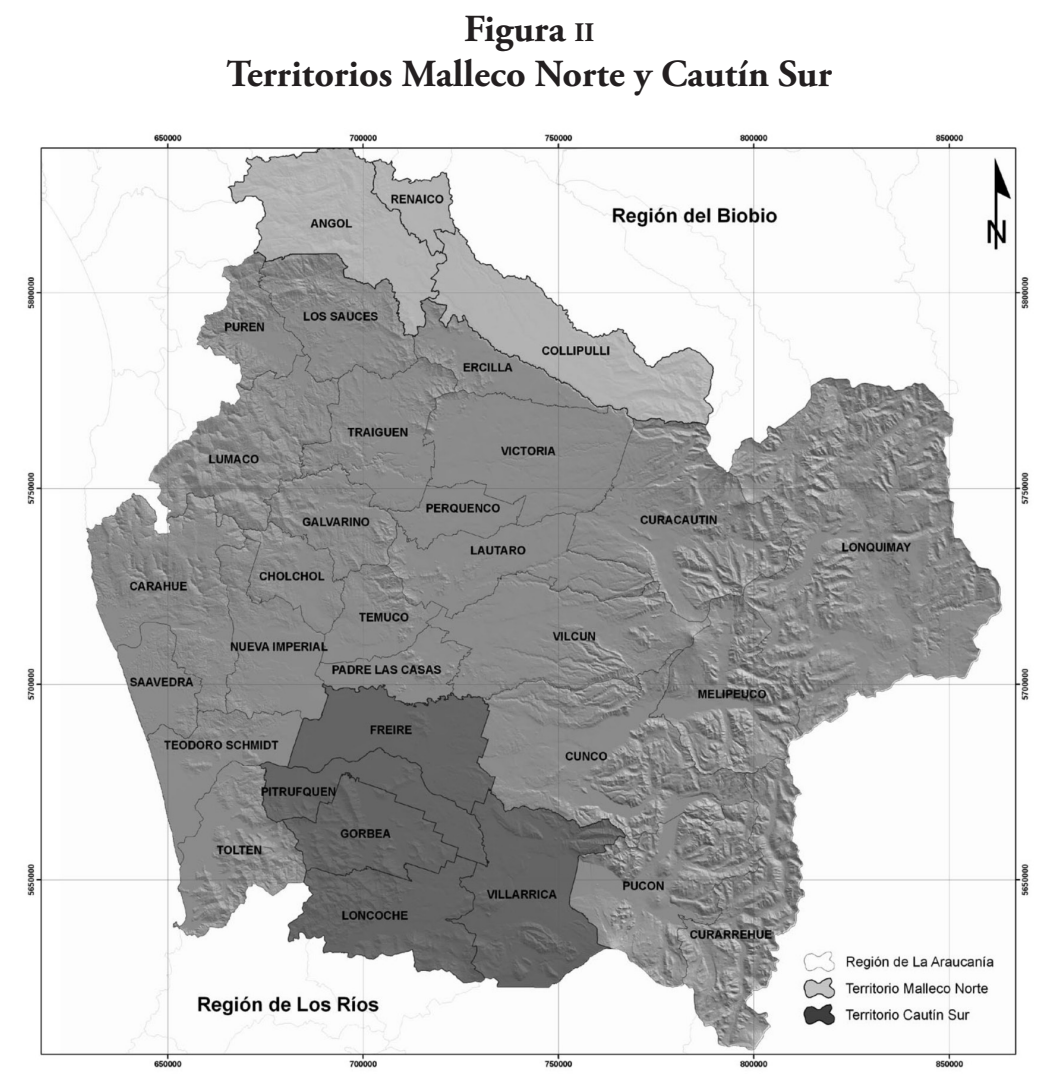

Fuente: Elaboración propia.

VBPP, seguida de la leche (18.2\%); donde la fruta queda relegada a sólo 5.9 por ciento.

El incremento de la actividad frutícola en la región también se confirma con los resultados del VII Censo nacional Agropecuario y Forestal (INE, 2007), el cual indica que la región representa 3.8\% de la superficie total del país (12.000 hectáreas), observándose que la producción de arándanos es seis veces mayor a la de la última década, lo que representa $14 \%$ del total plantado a nivel nacional. Igualmente sucede con el avellano europeo, que pasa de dos a 995 hectáreas, aportando 19\% del total en el país.

Aunado a ello, el Valor Bruto de Producción Primaria regional de fruta (odepa, 2007) asciende a la suma de 8.760 pesos. Por su parte la proyección para el 2014 muestra cambios muy significativos en la geografía económica de la fruta, por lo que se espera un valor para el 2014 cercano a 55.950 pesos (figura IV). Esto quiere decir que la región se está constituyendo en una exportadora de commodities. Al respecto, Katz (2000) plantea que las ramas manufactureras procesadoras de recursos 


\section{Figura III}

Valores brutos de producción primaria de los sectores productivos de La Araucanía para el 2006 y su proyección al 2014

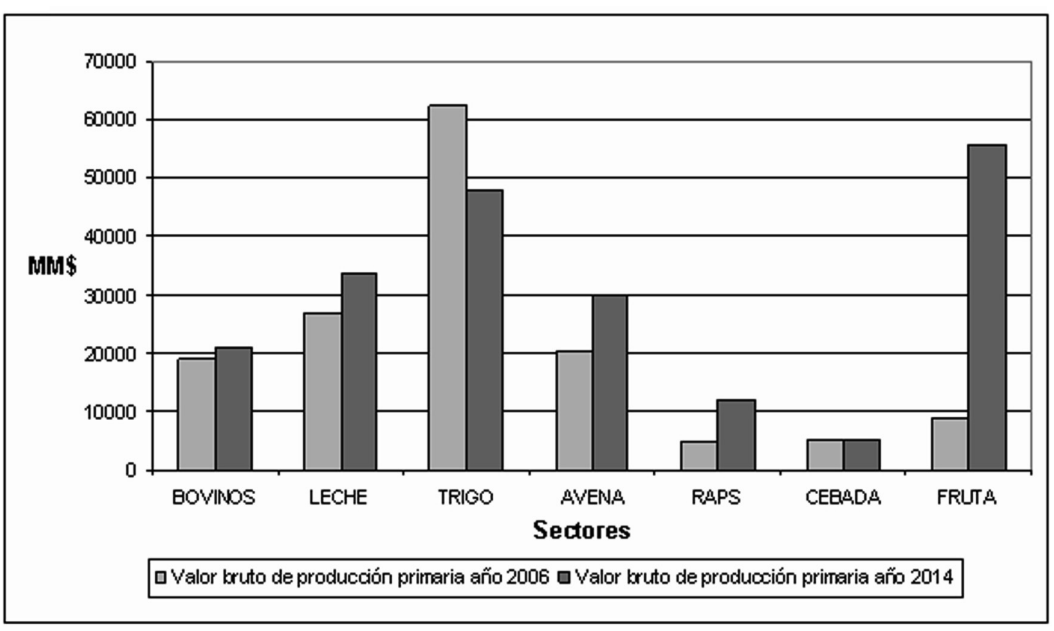

Fuente: Elaboración propia con base en datos de ODEPA (2007).

naturales, productoras de commodities, han logrado un mejor desempeño relativo a lo largo de los últimos veinte años, produciéndose a partir de ellas una transformación en los países de la región que los ha convertido en pocos años en fuertes exportadores de commodities.

Para el caso del territorio Malleco Norte, el Censo Agropecuario 2007 indica que cuenta con 2.835 hectáreas de frutales, equivalentes a 23.6\% de la superficie plantada a nivel regional, la cual según el modelo de aptitud podría aumentar en $23 \%$. Por su parte, el territorio Cautín Sur tiene 3.464 hectáreas de frutales equivalentes a $28.8 \%$ de la superficie regional, y las proyecciones del modelo desarrollado indican que podría, a lo menos, cuadruplicar su superficie, esta situación se explica por la mayor cantidad de superficie disponible apta para ser utilizada.

Uno de los elementos que ha permitido potenciar este rubro en la región lo constituye el riego, cuyo porcentaje de explotaciones regadas a nivel regional aumentó de 6 a 18\% entre los dos últimos censos. Además de lo anterior, existe un aumento del área regada por sistemas tecnificados, como mecánico mayor (aspersión tradicional, carrete o pivote), que en el último decenio prácticamente duplica su uso, alcanzando las 12.333 hectáreas. Por su parte, si se considera el volumen estimado total de fruta fresca procesada en la región, se calcula que se generan alrededor de 1300 empleos temporales, de los cuales, al igual que en el caso de la industria de congelados, la mayor proporción es ocupada por mujeres (Seremi de Agricultura, 2007). 


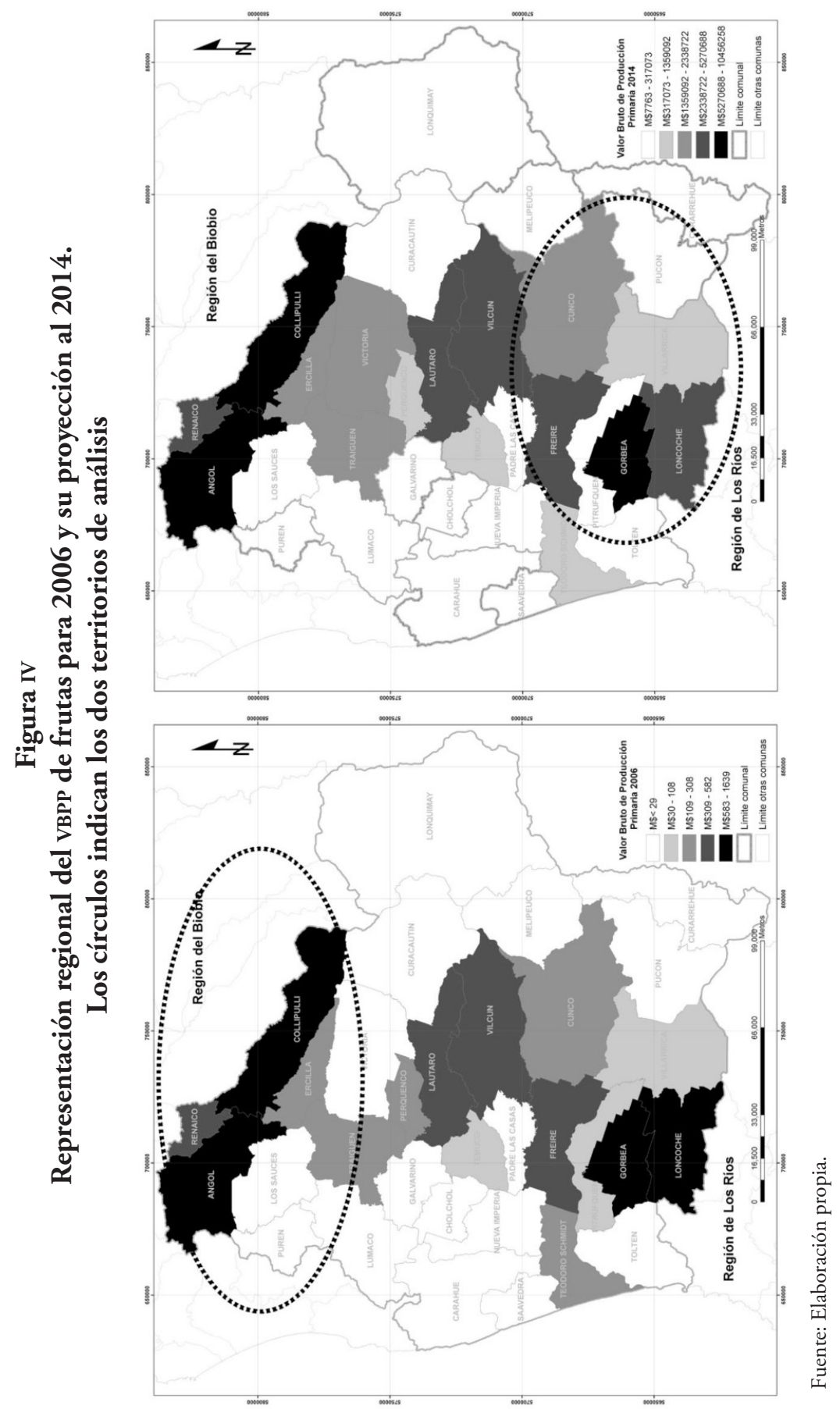


En este contexto, es importante mencionar que existen otros espacios de la región donde es posible expandir la actividad, siempre que en ellos se potencien las inversiones, elemento principal en el inicio de cualquier aglomeración productiva, y junto con ello existan instrumentos de fomento por parte del Estado que permitan dotar a estos sectores con aquellos factores de competitividad ( $\mathrm{I}+\mathrm{D})$, innovación, capital humano, inversión, conectividad favoreciendo los intereses territoriales (Del Castillo et al., 1994; Keating 1996, Wong-Gonzalez, 1999).

En este sentido, el vínculo entre el territorio y las cadenas productivas implican una serie de procesos en los que intervienen diferentes actores, quienes propician una red de relaciones y llevan a cabo una serie de acciones que permiten realizar una actividad específica en un espacio territorial determinado (Chavarría et al., 2002; Delgado et al., 2003).

Los datos anteriores, junto al análisis de innovación, capital humano e institucionalidad local, permiten completar las imágenes de un territorio con características productivas, económicas y sociales que permitiría mejorar la competitividad territorial de La Araucanía. En este sentido, nos encontramos inmersos en una economía globalizada, donde las regiones son un factor emergente de desarrollo frente a los estados y las ciudades, lo cual hace necesario tener una visión estratégica del territorio que permita aplicar en él políticas de ordenación, cuyo objetivo sea posicionarse mejor en un mundo competitivo (Hernández y Collado, 2004: 25).

Aunado a ello, si bien la competitividad tiene un sentido estrictamente económico, es necesario plantearla en un sentido más amplio: un territorio adquiere carácter competitivo si puede afrontar la competencia del mercado y garantizar al mismo tiempo la viabilidad medioambiental, económica, social y cultural, aplicando lógicas de red y de articulación interterritorial (Leader, 1999; IICA, 2003).

La nueva configuración territorial que presenta la región en torno a la actividad frutícola, no está ajena a las condiciones intrínsecas que tiene el territorio. En este sentido, es importante indicar que en la medida que aparecen rubros emergentes en la región, necesitan espacio en la matriz productiva regional. Esto trae como consecuencia una presión por la sostenibilidad del recurso suelo, el cual junto con la hidrografía y las áreas de riego se constituyen como agentes articuladores y detonantes de una serie de factores económicos, territoriales y políticos que intervienen en La Araucanía.

Esto ha dado paso a reconocer que junto con la competitividad territorial existe un capital territorial que posibilita el surgimiento de nuevos territorios. Por su parte, Delgado et al. (2003) y Canto (2000) señalan que el capital territorial identifica los elementos del territorio, de carácter tanto material como inmaterial, que pueden constituir en ocasiones un 
activo o una dificultad. Todos estos elementos integran la riqueza del territorio (actividades, paisajes, patrimonio, conocimientos técnicos, entre otros).

Para lograr consolidar a La Araucanía en este rubro se hace necesario incorporar experiencias de otras regiones, es por ello que se ha llegado a plantear que en la nueva economía global sólo pueden competir los territorios que aprenden, es decir, aquellos capaces de adaptarse a las transformaciones de la estructura productiva mundial, con base en el conocimiento (ampliamente entendido) y su aplicación al sector terciario avanzado, a la industria de alta tecnología y a la agricultura comercial (Fernández y Vigil, 2007; Lira, 2005).

En este sentido, las regiones que aprenden son aquellas que mejor enfrentan el juego globalizador y hacen de su capacidad de aprendizaje una condición esencial, que son capaces de resolver problemas, de experimentar nuevos enfoques, de aprovechar su propia experiencia para aprender de las experiencias de otras organizaciones (benchmarking) y de transmitir rápida y eficazmente el conocimiento a toda su estructura. La incorporación de estos elementos constituye un desafío importante para La Araucanía si se plantea ser competitiva en la actividad frutícola (Gatica, 2008; Sforzi, 2006).

De esta manera, la nueva generación de políticas de desarrollo se propone lograr que las ciudades y regiones se conviertan en territorios cada vez más competitivos y que resulten más atractivos para las inversiones y localizaciones de las empresas. Por ello, las estrategias y políticas económicas de las ciudades y regiones tienden a converger con las estrategias y las políticas de las empresas. El efecto combinado de las acciones de las empresas y de los territorios estimula los procesos de desarrollo económico y transforman la geografía económica (Vázquez, 2007; Rimisp, 2003).

La tendencia del aumento de la superficie destinada a la fruticultura desde 1965 a la fecha en La Araucanía permite identificar flujos económicos sobre el territorio, los cuales están dados por una nueva estructura del valor del producto bruto primario agropecuario regional, lo cual se relaciona con el nuevo escenario que se ha configurado para la política y estrategias de fomento exportador. La Araucanía ha estado incubando variados proyectos que dan cuenta de que los emprendimientos - basados en innovación, cumplimientos de estándares de calidad en procesos y productos, y en la exploración asociada de nuevos mercados, principalmente-, se orientan hacia una transformación que dará lugar a ajustes de variada magnitud en algunos territorios y cadenas de valor.

$\mathrm{Al}$ respecto Boisier (2006) plantea que la globalización empuja cambios profundos en la geografía política y económica, modificando la forma de competir. La competencia internacional por mercados, capital y tecnolo- 
gía deja de estar asociada únicamente con los países y comienza a asociarse con las ciudades y regiones. En efecto, todos los territorios compiten entre sí por los mismos elementos, en rigor, son directa e indirectamente competidores y competitivos.

\section{Conclusiones}

Los territorios identificados Malleco Norte y Cautín Sur abarcan 23\% de la superficie regional y en ellos se concentran $52.5 \%$ de las plantaciones de frutales. En estos espacios es posible expandir aún más la superficie plantada debido a características intrínsecas que presenta el territorio. Si a ello sumamos el efecto del cambio climático, estamos en presencia de una nueva tendencia en la configuración de la matriz productiva regional.

Para el caso de los territorios definidos es importante mejorar algunas brechas: en el caso de Malleco Norte, fortalecer el capital humano ya que el actual presenta baja especialización; incentivar la inversión privada, si bien ésta existe y ha permitido el desarrollo de la actividad, es necesario potenciarla aún más. Para el caso de Cautín Sur es necesario generar una política que permita incentivar el mejoramiento de infraestructura y conectividad, así como promover el desarrollo de ciencia, tecnología e innovación. Estos elementos constituyen factores de competitividad que dichos territorios deben mejorar para lograr su consolidación.

La metodología utilizada en el trabajo permitió integrar métodos provenientes del análisis económico con el modelamiento espacial, aportando a una comprensión más detallada de las dinámicas territoriales que se presentan en la región, desde un enfoque geográfico-territorial.

El desafío de los actores público-privados de La Araucanía es reconocer que en nuestra región existe una tendencia a la incorporación de nuevos rubros que se inicia a mediados del siglo xx y que se manifiesta de manera específica a partir de 1975. Esto implica poner en funcionamiento políticas, estrategias y una institucionalidad orientada a generar flujos de productos hacia mercados extraterritoriales, lo que permitirá que los territorios se adapten mejor a las nuevas condiciones de mercado y contribuyan con ello a neutralizar los efectos de desigualdad de los procesos de integración. 


\section{Bibliografía}

ARdP (Agencia Regional de Desarrollo Productivo) (2008), Plan de mejoramiento de la competitividad fruticola. Informe final, Laboratorio de Planificación Territorial-Corparaucanía-Instituto Desarrollo Regional, Temuco.

Albuquerque, Francisco (2006), "Clusters, territorio y desarrollo empresarial: Diferentes modelos de organización productiva”, Cuarto Taller de la Red de Proyectos de Integración Productiva Fondo Multilateral de Inversiones (MIF/FOMIN)-Banco Interamericano de Desarrollo, San José de Costa Rica, 10-12 julio.

Barton Jonathan, Robert Gwynne and Warwick Murray (2007), "Competition and co-operation in the semiperiphery: closer economic partnership and sectoral transformations in Chile and New Zealand", The Geographical Journal, 173 (3), Wiley, London, pp. 224-241.

Bengoa, José (1996), Historia del pueblo mapuche, Editorial Interamericana, Santiago de Chile.

Boisier, Sergio (2006), Imágenes en el espejo: aportes a la discusión sobre crecimiento y desarrollo territorial, Puerto de Palos, Santiago de Chile.

BCG (Boston Consulting Group) (2007), Estudio de competitividad en clusters de la economía chilena. Documento de referencia Fruticultura primaria. Parte 1, Consejo Nacional de Innovación y Competitividad, Santiago de Chile.

Canto, Consuelo (2000), "Nuevos conceptos y nuevos indicadores de competitividad territorial para las áreas rurales", Anales de Geografía de la Universidad Complutense, núm. 27, Universidad Complutense de Madrid, Madrid, pp. 69-84.

Costa-Filho, Alfredo (1997), "Inflexiones recientes en el análisis prospectivo: exigencias actuales de mega ajustes", en Javier Medina Vásquez y Edgar Ortegón (comps.), Prospectiva: Construcción social del futuro, Universidad del Valle-Instituto Latinoamericano y del Caribe de Planificación Económica y Social, Santiago de Cali, 7 p. 
Cuervo, Luis (2006), "Globalización y territorio", Serie Gestión Pública, núm. 56, Naciones Unidas-CEPAL, Santiago de Chile.

Chavarría, Hugo, Patricio Rojas y Sergio Sepúlveda (2002), Cuaderno Técnico $N^{\circ}$ 23. Competitividad: cadenas agroalimentarias y territorios rurales, Instituto Interamericano de Cooperación para la Agricultura, San José de Costa Rica.

Delgado, Carmen, Carmen Gil, Luis Hortelano y Juan Plaza (2003), "Turismo y desarrollo local en algunas comarcas de la montaña cantábrica: Recursos y planificación”, Cuadernos de turismo, 12, Ediciones de la Universidad de Murcia, Murcia, pp. 7-34.

Del Castillo, J., B. Barroeta, M. Bayón y E. Cordero (1994), Manual de Desarrollo Local, Departamento de Economía y Hacienda del Gobierno Vasco, Vitoria-Gasteiz.

Etcheverri, Rafael (2003), "Desarrollo territorial sostenible, enfoque territorial”, Sinopsis 2003, Área de Desarrollo Rural SostenibleInstituto Interamericano de Cooperación para la Agricultura, Santiago de Chile, pp. 1-11.

Fernández, Víctor, Belén Alfaro y Carina Davies (2009), “Aglomeraciones productivas y territorio: en busca de una manera más holística de entender sus contribuciones al desarrollo", Economía, Sociedady Territorio, IX (31), El Colegio Mexiquense A.c., Zinacantepec, pp. 629-680.

Fernández, Víctor y José Vigil (2007), “Clusters y desarrollo territorial: revisión teórica y desafíos metodológicos para América Latina”, Economía, Sociedad y Territorio, VI (24), El Colegio Mexiquense A.C., Zinacantepec, pp. 859-912.

Fonte, María (2006), Desarrollo rural e identidad cultural: Reflexiones teóricas y casos empíricos, Universidad de Nápoles "Federico II", Santiago de Chile.

Fujita, Masahisa y Paul Krugman (2004), "La nueva geografía económica: pasado, presente y futuro", Investigaciones Regionales, núm. 004, Asociación Española de Ciencia Regional, Alcalá de Henares, pp. 177-206. 
Friedman, Thomas (2006), La tierra es plana. Breve historia del mundo globalizado del siglo XXI, Ediciones Martínez Roca, Madrid.

Gatica, Francisco (2008), Redes y oportunidades de desarrollo. El caso de los circuitos económicos locales en el secano interior de la región del Biobio, Ediciones Universidad del Biobio, Concepción.

Gómez Orea, Domingo (2007), Ordenación territorial, Ediciones Mundi Prensa-Editorial Agrícola Española, Madrid.

Gómez, Montserrat y José Barredo (2006), Sistemas de información geográfica y evaluación multicriterio en la ordenación del territorio, Alfaomega, México.

Hernández, Nieves y Juan Carlos Collado (2004), "La estrategia territorial en Navarra: una experiencia de aplicación de los principios de planificación y desarrollo espacial sostenible europeos a nivel regional", V Congreso de Economía de Navarra, 20 y 21 de noviembre de 2004, Pamplona.

IICA (Instituto Interamericano de Cooperación para la Agricultura) (2003), "Territorios rurales, competitividad y desarrollo", Cuaderno Técnico, núm. 23, IICA, San José de Costa Rica.

IGM (Instituto Geográfico Militar) (1986), Geografía IX Región de La Araucanía, Ediciones del Instituto Geográfico Militar de Chile, Santiago de Chile.

INE (Instituto Nacional de Estadísticas) (2007), VII Censo nacional agropecuario y forestal. Documento Resultados preliminares, Ediciones del Ine, Santiago de Chile.

Jara E. y Valeria N. (1987), “Toltén y la ocupación del litoral”, Seminario Reflexiones del borde costero de La Araucanía. Universidad de la Frontera, 20-22 de agosto, Temuco.

Katz, Jorge (2000), Reformas estructurales, productividad y conducta tecnológica en América Latina, División de Desarrollo Productivo y Empresarial-Comisión Económica para América Latina y el Caribe, Santiago de Chile. 
Keating, Michael (1996), Naciones contra el Estado. El nacionalismo de Cataluña, Quebec y Escocia, Ariel Ciencia Política, Barcelona.

Leader (Liaisons entre activités de Developement de L'Economie Rural) (1999), La competitividad territorial. Construir una estrategia de desarrollo territorial con base en la experiencia de Leader, Fasciculo 1. Comisión Europea-Dirección General de Agricultura, Ecuador.

Lira, Iván (2005), "Desarrollo económico local y competitividad territorial en América Latina", Revista de la CEPAL, núm. 85, Santiago de Chile, pp. 81-100.

odepa (Oficina de Estudios y Políticas Agrarias) (2007), Informe situación de la agroindustria en la región de La Araucanía, Ediciones ODEPA Chile, Temuco.

odepa (Oficina de Estudios y Políticas Agrarias) (2009), Situación de la industria de la fruta fresca en la temporada 2008/2009, Publicación de la Oficina de Estudios y Políticas Agrarias-odepa, Santiago de Chile.

Pacheco, Diego (2001), Visiones sobre la territorialidad y el desarrollo rural, Taller de Iniciativas en Estudios Rurales y Reforma Agraria, La Paz, p. 25.

Peña, Fernando y Miguel Escalona (2009), "Expansión urbana en la intercomuna Araucanía Centro. Alteraciones sobre las áreas rurales", en R. Hidalgo y F. Arenas (eds.), Chile del país urbano al pais metropolitano, Ediciones Pontificia Universidad Católica de Chile, Santiago de Chile, pp. 389-398.

Pike, Andi, Kean Birch, Danny MacKinnon y Robert McMaster (2009), "A geographical political economy of evolution in economic geography”, Journal of Economic Geography, 85 (2), Oxford University Press, Malden, pp. 175-182.

Ranaboldo, Claudia y Alexander Schetjmann (2009), El valor del patrimonio cultural. Territorios rurales, experiencias y proyecciones latinoamericanas, Rimisp Editores, Santiago de Chile. 
Rimisp (Centro Latinoamericano para el Desarrollo Rural) (2003), El desarrollo territorial rural, Rimisp Editores, Santiago de Chile.

Rosenblitt, Jaime y Ricardo Naser (2005), Entre el mar y Nahuelbuta: Historia del asentamiento humano en Arauco, Archivo ChileCentro estudios Miguel Enríquez, Concepción.

Santé-Riveira, Inés y Crecente-Maseda, Rafael (2005), "Evaluación de métodos para la obtención de mapas continuos de aptitud para usos agroforestales", GeoFocus, núm. 5, Madrid, Grupo de Tecnologías de la Información Geográfica-Asociación de Geógrafos Española, Madrid, pp. 40-68.

Seremi de Agricultura (Secretaría Regional Ministerial de Agricultura) (2007), "Situación actual del desarrollo productivo en La Araucanía”, Informe semestral, Temuco, p. 50.

Schetjmann, Alexander y Julio Berdegue (2004), Desarrollo territorial rural, Centro Latinoamericano para el Desarrollo Rural-Rimisp, Santiago de Chile.

Sforzi, Fabio (2006), “El distrito industrial y el 'viraje territorial' en el análisis del cambio económico”, Economía Industrial, Universidad de Parma, Parma, pp. 37-42

Vázquez-Barquero, Antonio (2006), "Surgimiento y transformación de clusters y milieus en los procesos de desarrollo”, EURE, 32 (95), Pontíficia Universidad Católica de Chile, Santiago de Chile, pp. 75-92.

Vázquez-Barquero, Antonio (2007), "Desarrollo endógeno. Teorías y políticas de desarrollo territorial”, Investigaciones Regionales, núm. 11, Asociación Española de Ciencia Regional, Madrid, pp. 183-210.

Wong-González Pablo (1999), "Globalización y virtualización de la economía: impactos territoriales”, ponencia presentada en el V Seminario de la Red Iberoamericana de Investigadores sobre Globalización y Territorio, convocada por la Red Iberoamericana de Investigadores sobre Globalización y Territorio y la Universidad Autónoma del Estado de México, 21-24 de septiembre, Toluca. 
Recibido: 24 de marzo de 2010.

Reenviado: 2 de mayo de 2012. Aceptado: 26 de diciembre de 2012.

Miguel Antonio Escalona Ulloa. Es magíster en planificación y gestión territorial por la Universidad Católica de Temuco, Chile. Actualmente es profesor asistente de la Escuela de Ciencias Ambientales de la Facultad de Recursos Naturales e investigador del Laboratorio de Planificación Territorial de la misma universidad. Su línea de investigación actual es la planificación territorial y las dinámicas económico-territoriales. Entre sus últimas publicaciones destacan, en coautoría: "Evaluación ambiental estratégica: Instrumento para la planificación territorial urbana”, Revista Urbano, 15 (25), Universidad del Bío Bío, Concepción, pp. 17-30 (2012); "Oferta turística y perfil sociocultural de los empresarios turísticos del Borde Costero de La Araucanía, Chile", Perspectivas en Turismo, vol. 21, Centro de Investigaciones y Estudios Turísticos, Buenos Aires, pp. 306321 (2012); "Análisis del sistema turístico en el destino Sietelagos, Panguipulli, región de Los Ríos. Aportes a la planificación y desarrollo territorial”, Revista Chilena de Estudios Regionales, vol. 2, Red Sinergía Regional, Agrupación de Universidades Regionales, Chile, pp. 64-77 (2011); "Cambio de uso del suelo en los geosistemas de la cuenca costera del Río Boroa (Chile) entre 1994 y 2004”, Revista de la Facultad de Ciencias Agrarias de la Universidad Nacional de Cuyo, 43 (2), uncuyo, Mendoza, pp. 1-20 (2011).

Fernando Andrés Peńa Cortés. Es doctor en ciencias ambientales por la Universidad de Concepción, Chile. Actualmente es profesor-investigador, titular de la Escuela de Ciencias Ambientales de la Facultad de Recursos Naturales y coordinador del Laboratorio de Planificación Territorial de la misma universidad. Su línea de investigación actual es la planificación territorial. Entre sus últimas publicaciones destacan, en coautoría: "A study of the geographic distribution of swamp forest in the coastal zone of the Araucanía region, Chile", Applied Geography, 31, Universidad Católica de Temuco, Temuco, pp. 545-555 (2011); "Gestión de la emergencia ante eventos de inundación por Tsunami en Chile: el caso de Puerto Saavedra”, Revista de Geografía Norte Grande, Pontificia Universidad de Chile, Santiago de Chile, 47, pp. 63-80 (2010); "Cambio de uso del suelo en los geosistemas de la cuenca costera del Río Boroa (Chile) entre 1994 y 2004", Revista de la Facultad de Ciencias Agrarias de la Universidad Nacional de Cuyo, 43 (2), uncuyo, Mendoza, pp. 1-20 (2010). 
Gonzalo Daniel Rebolledo Castro. Es magíster en ciencias con mención en producción, manejo y conservación de recursos naturales por la Universidad de Los Lagos, Chile. Actualmente es profesor asistente de la Escuela de Ciencias Ambientales de la Facultad de Recursos Naturales e investigador del Laboratorio de Planificación Territorial de la Universidad Católica de Temuco. Su área de trabajo actual se centra en la representación, análisis y modelación de geoinformación en aplicaciones de ecología espacial, paisaje y territorio. Entre sus últimas publicaciones destacan, en coautoria: "Cambio de uso del suelo en los geosistemas de la cuenca costera del Río Boroa (Chile) entre 1994 y 2004", Revista de la Facultad de Ciencias Agrarias de la Universidad Nacional de Cuyo, 43(2), uncuyo, Mendoza, pp. 1-20 (2011); "Análisis de la estructura y organización de un paisaje forestal del sur de Chile", Gestión Ambiental, 19, CEA ediciones, Chile, pp. 47-66 (2010); "Uso y selección de hábitat por mamíferos carnívoros y herbívoros en bosque nativo y plantaciones forestales del sur de Chile", Gestión Ambiental, 19, ceA ediciones, Chile, pp. 33-46 (2010); "Diagnóstico del sistema turístico en la cuenca del Lago Ranco", Revista Lider, Universidad Católica de Temuco, Chile, 16, pp. 159-172 (2010).

Pío Iván Antonio Basso Aldea. Licenciado en ciencias agronómicas por la Universidad Austral de Chile. Actualmente se desempeña como profesional de apoyo en la Secretaría Regional de Agricultura Región de La Araucanía. Sus áreas de trabajo se enfocan en la economía y desarrollo territorial. 